\title{
AMBIGUEDAD DE LOS INDICADORES Y LA DIAGNOSIS DE PROCESOS
}

\author{
Hugo Zemelman M. \\ El Colegio de México
}

\begin{abstract}
A LAS SOCIEDADES de finales del siglo XX afectan problemas similares tales como el deterioro del medio ambiente, la manipulación de las empresas transnacionales, el armamentismo, la ausencia de una efectiva participación en las decisiones, desempleo, inflación. Los datos oficiales por lo general ocultan la realidad de los fenómenos que deben preocuparnos, lo que nos lleva a centrar nuestra atención en dos problemas: a) cuáles son los desafíos que enfrentan las sociedades latinoamericanas; $y$ b) qué implicaciones tienen para el problema de los indicadores.
\end{abstract}

\section{Desafios y riesgos}

El título en sí mismo es ambiguo. Debemos por lo tanto, aclarar el significado de cada una de estas palabras. Desafío significa reconocer la necesidad imperiosa de desarrollar una respuesta ante una situación inaceptable; pero el desafío puede reconocer varias respuestas siendo algunas de ellas viables y otras no. Lo anterior implica un riesgo que consiste en equivocarse en el diagnóstico de las alternativas de desarrollo y en la consiguiente definición de políticas.

¿Cuáles son los desafíos en nuestros países hoy en día? Habría que comenzar por afirmar que desconocemos si los riesgos que vislumbramos son propios de una situación histórica o, más bien, son coyunturales. Sin aventurar afirmaciones de carácter sustantivo (pues para ello habría que hacer toda una investigación concreta en diferentes países), podríamos señalar los siguientes desafíos para los países latinoamericanos, con excepción de Cuba y Nicaragua que merecen un análisis separado, aunque como es evidente compartan algunos de ellos. Distinguiremos entre los aspectos económicos, políticos y culturales. 


\section{Aspectos económicos}

Los estudios sobre la inserción de los países latinoamericanos en la nueva división internacional del trabajo, marcan los siguientes rasgos:

a) apertura de los mercados nacionales a la inversión internacional mediante una politica de liberalización, o de desprotección de la actividad productora interna, que ha significado un rompimiento del clásico modelo desarrollista-proteccionista prohijado por la Comisión Económica para América Latina;

b) tendencia a reemplazar a la burguesía manufacturera nacional por las empresas multinacionales;

c) transformación de esta burguesía en importadora-exportadora;

d) robustecimiento de la actividad de la fracción financiera a través de su alianza con los grandes bancos internacionales;

e) orientación hacia una política que aproveche las ventajas comparativas para ganar penetración en el mercado internacional;

f) predominio de los modelos monetaristas antiinflacionistas sobre los modelos de crecimiento económico;

g) retraimiento del Estado de su papel de interventor en la economía procurando que este rol lo asuma el sector privado;

h) tendencia en las estrategias de desarrollo de relegar a un plano secundario las metas de empleo y distribución del ingreso en forma de contribuir a la creación de un mercado interno;

i) predominio de los préstamos a corto plazo orientados al pago de la deuda externa sobre la inversión productiva a largo plazo; etc.

La debilidad de las economías nacionales para conquistar nuevos márgenes de crecimientos, más allá del que ofrecía la política de sustitución de importaciones (que fuera la estrategia dominante en la década de los cuarenta hasta comienzos de los setenta) determina una crisis para la dominación burguesa vigente que no puede ser resuelta por los medios populistas.

Estos modelos no hacen más que precipitar la crisis al desatar nuevas presiones, provenientes de los sectores populares (movimiento obrero organizado, capas medias y más tardíamente los sectores campesinos), sobre la capacidad de acumulación manteniendo altas tasas de ganancia (las que nunca fueron muy altas). Se hace cada vez más evidente la incompatibilidad entre la acumulación y una política redistributiva propia de los sistemas políticos demo-liberales o populistas.

La necesidad de nuevos mercados del capitalismo desarrollado y la búsqueda de condiciones de producción menos costosas que en los países de origen, concurren como factores a explicar el cambio de rumbo en las estrategias de desarrollo que sumariamente caracterizábamos. Importa destacar, que uno de los factores que determina el poco dinamismo de las burguesías criollas es su tecnología obsoleta que le cierra posibilidades de acceso competitivo al mercado internacional, considerando la tendencia a la concentración y centralización que experimenta el capital internacional. 
Del cuadro anterior se desprenden algunos desafíos como los siguientes:

a) la apertura de los mercados nacionales para la transferencia de capitales (producto en parte de la sobreacumulación en los paises industrializados) se acompaña por lo general de un deterioro en los términos del intercambio que contribuye a agravar la crisis de financiamiento;

b) la excesiva dependencia de las economías abiertas a los grandes bancos internacionales incrementa su deuda externa de corto plazo y a tasas altas de interés;

c) la gradual transnacionalización cuando no directa destrucción de la industria manufacturera determina un predominio del capital financiero con fines mercantiles, usurarios y especulativos.

La inserción de las economías latinoamericanas en el mercado internacional, vía capital financiero, determina grosso modo un proceso de liquidación de las economías nacionales que se expresa en un estancamiento de los índices de producción industrial, en un deterioro en el valor agregado de sus exportaciones y en un incremento de las importaciones con el consiguiente déficit en la balanza de pagos, que es el costo para facilitar la entrada del capital financiero, estrategia económica que se acompaña de gobiemos fuertes los cuales, paradójicamente, asumen el carácter de regímenes "nacionalistas".

En efecto, el esquema de una economía abierta al mantener una tasa de desempleo promedio superior al $8 \%$ de la población económicamente activa sin considerar la desocupación disfrazada que es absorbida por el sector terciario, y una tasa de quiebras de empresas medianas y pequeñas (como son muy ilustrativos los casos de Argentina y Chile: en Chile el número de quiebras en 1980 es 15 veces mayor que en 1975), no puede sostenerse sino a través de una política represiva. La penetración del capital financiero, como la modalidad dominante en la inserción de A.L. en la nueva división internacional del trabajo, se expresa políticamente en los Estados militares.

Las implicaciones de lo descrito son evidentes:

a) desempleo estructural;

b) distribución regresiva del ingreso;

c) agudización en las relaciones de dependencia;

d) restricciones a los organismos de la sociedad civil;

e) inseguridad personal;

f) marginación social y politica;

g) falta de expectativas para un desarrollo personal, excepción de los grupos económicamente dominantes.

Frente a este cuadro socio-político la alternativa es la eliminación del modelo oligárquico-financiero cuya expresión política es la dominación burocrática-militar. Desde el punto de vista de la claboración de políticas se plantean dos cuestiones a resolver: i) la interpretación de los síntomas (indicadores) en que se manifiesta el cuadro descrito; y ii) la elaboración de la información que nos permita 
tomar conciencia de las potencialidades de cambio que se contienen en la situación.

Respecto al punto $\mathbf{i}$, surgen problemas estrechamente relacionados con el carácter no compensatorio de los indicadores considerados como sistema. Nos encontramos con contradicciones más o menos burdas que exigen ser resueltas en función de opciones ideológicas. Se podría ejemplificar lo dicho en relaciones del siguiente tipo:

1. Incremento en el GN con đesempleo estructural de la población económicamente activa;

2. desempleo sin subsidio de cesantía; desempleo con subsidio de cesantía;

3. incremento de las importaciones con deterioro de los términos de intercambio;

4. cambio en la estructura de las exportaciones (diversificación) pero con falta de desarrollo tecnológico que mejore la competitividad;

5. incremento en la oferta de servicios (de salud, educación, alimentos, etc.) con exigencia de auto-financiamiento;

6. auto-financiamiento con concentración del ingreso;

7. capacidad de pago con liquidez internacional e infraestructura industrial deficitaria; etc.

Si pensamos en la situación 1 es obvio que el significado que tenga el indicador GN estará determinado por la tasa de cesantía, toda vez que sus efectos sociales (más allá de su puro perfil numérico) estarán mediados por el acceso de la población que será en función del desempleo; pero, a su vez, la tasa de desempleo está condicionado en su impacto sobre el mercado interno por la existencia o ausencia de una política de subsidios. Es obvio que ser cesante con subsidio o sin este conforma una situación socio-política completamente distinta. El mismo subsidio asume un carácter u otro según lo sea para pagar desempleados estructurales que si se emplea para subsidiar cesantes estacionales o cíclicos.

Las situaciones 3 y 4 sirven para ilustrar otro tipo de situaciones problemáticas en que se reflejan las relaciones no compensadas de los posibles indicadores. $\mathrm{El}$ incremento de las importaciones (al margen de cualquier consideración relativa a su composición, que daría lugar a otra situación problemática) asume diferentes significados según tengan lugar en un contexto de deterioro o no en las relaciones de intercambio; las cuales pueden ser función estricta de la disminución u oscilación en el precio del o de los productos de exportación (caso de los países monoexportadores es el más claro), o estar relacionada con un insuficiente desarrollo tecnológico que impide penetrar y conservar mercados de colocación en condiciones de competencia.

Por su parte, los indicadores de desarrollo tecnológico aparecen cumpliendo una multifunción ya que pudiendo contribuir a incrementar la productividad, volumen y calidad de la oferta no significa que produzcan un efecto social posible de establecerse en forma directa, pues la exigencia de autofinanciamiento de los servicios impide el acceso a ellos de cierta proporción de la población. 
De otra parte, el desarrollo tecnológico, que está vinculado a las actividades agrícolas de exportación, tiene un significado como indicador de desarrollo muy diferente a si está vinculado a actividades económicas orientadas a crear las bases de autosustentación del desarrollo.

El insuficiente desarrollo económico medido a través de la relación entre PNB y capacidad de pago externo asume un contenido muy diferente en un contexto de liquidez internacional o a la inversa, de falta de circulación en el mercado de capitales. Así, el monto de la deuda externa no representa un obstáculo para nuevos préstamos en situaciones en que el capital requiere sobrevalorizarse en economías periféricas, que si, por el contrario, no se producen situaciones como esa. Lo que sostenemos es que la función y significado de los indicadores mediante los cuales se puede interpretar un problema depende de la naturaleza problemática de las situaciones; las cuales serán un reflejo de la complejidad que ofrece la realidad, por lo que en una medida importante son imprevisibles. Por eso consideramos que no tiene sentido elaborar listas de indicadores normativos que fijan de alguna manera una meta deseable, ya que el problema de fondo es reconocer esas situaciones problemáticas como las ejemplificábamos, en el aspecto económico, en forma desde luego simplificada.

El significado de un indicador es función de una situación concreta, a veces compleja y difícil de reconstruir. Si lo es considerando exclusivamente los aspectos económicos, mucho mayor complejidad adquiere cuando pensamos en incluir otros aspectos de la realidad (políticos, culturales, sico-sociales). Si nos detenemos a analizar indicadores económicos ilustrativos, como los mencionados, podemos fácilmente darnos cuenta de que expresan una dinámica no restringida al plano de lo económico, pues, además reflejan una dinámica política que conforma un estilo de desarrollo (desarrollo industrial - desarrollo agricola; economía orientada hacia el mercado interno o hacia la exportación; política de inversión y de gasto público; política de ingreso; patrón de desarrollo tecnológico; etc.). Fuerzas que se expresan en el plano político, o que reconocen en este plano de la realidad condiciones que las influyen, como ser concentración del poder económico en determinados grupos de presión; grado de cohesión ideológica de estos grupos; acceso a los centros de decisión en el aparato institucional del Estado (lo que dependerá, por ejemplo, del grado de descentralización de esta estructura, etc.); relaciones de las diferentes fuerzas sociales con la sociedad civil (sindicatos, partidos), etc. Esta complejidad de elementos confluye en situaciones problemáticas cuya concreción está determinada por el modo como los diferentes elementos componentes de la realidad se articulån entre sí.

Estas situaciones problemáticas son las que hay que reconocer considerando su mutabilidad y variedad a través de un listado de indicadores. Es necesario reconstruir las situaciones no mediante el expediente de darle un contenido al indicador (cualquiera sea éste), a través de su definición operacional, sino transformándolo en elemento de una articulación que abarque el conjunto de las grandes áreas de la realidad (económica, política, cultural y sico-social). Ello supone que 
por sobre el listado predomine una lógica de reconstrucción de la realidad problematizada en funcióri de una base en referencia que cumpla la función de eje teórico (hipótesis) o social (problema).

De conformidad con lo anterior, no se trata tanto de inventar nuevos indicadores, o de forjar contra-indicadores a los ya estandarizados por las agencias oficiales, como de diseñar una lógica que permita reconocer las situaciones problemáticas específicas que determinan el significado que, en un momento, asumen el o los indicadores.

Lo anterior valga como advertencia metodológica, ya que el reconocimiento de estas situaciones concretas es la función de los indicadores, aunque, simultáneamente, los indicadores están conformados en su contenido por la situación que se trata de reconocer a través de ellos. De ahí que la exigencia de pensar a la realidad desde una exigencia de articulación lleva necesariamente a tener que especificar el significado de cada indicador en función de la articulación que contribuye a reconstruir. El aparente círculo cerrado se rompe desde el momento que la articulación exige que cada indicador sea leído desde el conjunto, tanto como el conjunto desde cada uno en particular. ${ }^{1}$

\section{Aspectos politicos y culturales}

La superación de una situación como la descrita implica enfrentarse también con aspectos de la vida política que son de gran complejidad. No se trata solamente de que la coyuntura actual se caracterice por lo que hemos llamado modelo oligárquico-financiero-burocrático-militar, ya que cualquier altẹnativa supone, además de cambiar las situaciones que hemos ejemplificado en las páginas anteriores, tener que pensar en los obstáculos políticos y culturales que dificultan la viabilidad de una alternativa. Nuevamente el problema no es sólo determinar la posibilidad objetiva de una línea de acción sino la interpretación adecuada de los síntomas. El mismo ejercicio que hacíamos para los aspectos económicos podemos repetirlo para los aspectos políticos y culturales.

Si pensamos que la alternativa debe serlo a los peligros de la transnacionalización de la economía, $o$, más concretamente, de la inserción en la división internacional del trabajo a través de la penetración del capital financiero, debe plantearse un diagnóstico socio-político que permita avanzar en el reconocimiento de lo que es viable. Es preciso definir las áreas problemáticas en las que hay que definir las políticas alternativas. Sin embargo, como cuando nos referíamos a los aspectos económicos tendremos que limitarnos a afirmaciones generales, aunque partiendo de procesos que afectan a los países latinoamericanos en diferentes formas e intensidades.

1 En el informe que hemos preparado para la investigación sobre indicadores El ColegioUNO hemos esbozado los lineamientos fundamentales de un planteamiento de esta naturaleza. (Trabajo de Próxima Publicación). 
Un primer riesgo que plantea el modelo econónico son sus efectos sobre la identidad nacional. Es claro que la penetración de las empresas transnacionales se acompaña de una estandarización de los productos que con el tiempo modifica los patrones culturales de la población. La tendencia a la homogeneización (a reserva de que esta constituye una afirmación que requiere de estudios empíricos concretos) se puede traducir en comportamientos, de movilidad enajenantes del individuo. Un ejemplo servirá para ilustrar lo que decimos.

En los barrios populares de Santiago de Chile se puede apreciar una reproducción de la oferta de bienes de consumo no esenciales que es muy semejante a la que puede observarse en los barrios de altos ingresos, variando solamente la calidad de los bienes de acuerdo con el nivel socioeconómico. Ello crea una expectativa de consumismo cuyo efecto es múltiple.

No es poco frecuente encontrarse con pobladores (especialmente mujeres) que estando cesantes $y$, en consecuencia, sin ingresos son partidarios del régimen militar con base en argumentos ideológicos como el siguiente: "ahora, en comparación con lo que pasaba durante el régimen de la Unidad Popular, no hay desabastecimiento (acotemos que éste fue provocado por los comerciantes como una de las medidas de hostigamiento en contra del Gobierno); hay que comparar y para ello no hay que formarse en largas filas". Es interesante observar que respuestas de esta naturaleza las dan personas que no compran por carecer de ingresos, lo que puede interpretarse como una expectativa de movilidad subjetiva que se corresponde con una falta de conciencia política muy aguda. Un efecto potencial de este consumismo es que se crean las condiciones para que los paises con los que las empresas productoras de los bienes ofertados se identifican vayan gradualmente transformándose en modelos de referencia, incluso para los sectores marginales. El consumismo no se limita, por consiguiente, sólo a producir efectos enajenantes en cuanto se refiere a la identidad nacional; también estimula un individualismo en el comportamiento que dificulta iniciativas solidarias que no tengan una relación directa con sus necesidades inmediatas. De todo lo cual se puede caracterizar una situación problemática en los siguientes términos.

i) acceso al consumo

Consumo ii) efectos del consumo sobre el nivel de vida -disponibilidad de ingreso monetario

-funcionamiento adecuado de los mecanismos de distribución

-el nivel de vida como mecanismo reproductivo biológico (comer, etc.)

-el nivel de vida como mecanismo reproductor social (estilo de vida)

-desarrollo de nuevas expectativas 
iii) efectos del consumo sobre las orientaciones valorativas

Cada uno de los elementos de esta itemización puede convertirse en un indicador. Sin embargo lo que específicamente significa cada ítem dependerá del conjunto que es el que nos proporciona la base para prevenir problemas y definir políticas alternativas. Examinemos algunas alternativas desde las más simples a las más complejas.

1. El monto del ingreso como indicador de acceso al consumo queda anulado por una mala distribución (para no hablar de una crisis en la producción); una red de distribución bien organizada en un sistema de mercados locales queda anulada como indicador de acceso al consumo como resultado de una distribución regresiva del ingreso, o bien por una excesiva concentración de la población en ciertos lugares;

2. el acceso al consumo adquiere significación específica según cumpla una función de reproducción (comer), que si cumpliera una función social e ideológica (tipo de comida, lugares de comer, etc.). En el primer caso, los componentes nutricionales son básicos para interpretar correctamente la función que cumple el acceso al consumo; mientras que en la segunda situación no revisten ninguna. Sin embargo el estilo de vida influye sobre la dieta alimenticia hasta el punto de impedir un cambio de ésta para una mayor calidad nutricional.

3. Lo anterior nos remite a tener que considerar al nivel de vida, en cuanto mecanismo que cumple una función social e ideológica, como expresión de un proyecto de sociedad identificable con determinados grupos sociales; o bien como la imposición del proyecto propio de otro grupo. De manera que el nivel de vida, en esta acepción, puede ser expresión tanto de la capacidad de un grupo social para definir políticas, como también reflejar el sometimiento ideológico de un grupo respecto de otro. Es la ambivalencia que ofrecen los patrones de vida los cuales son función de una concepción de vida. Así, la habitación, el vestuario, etc. están mediados de su función básica de reproducción para transformarse en indicadores de proyectos de vida que resultan de una cierta visión de la realidad;

4. Pero la situación problemática que tratamos de construir a manera de ilustración se puede también rastrear sin necesidad de relacionar ítems. Se puede reconstruir al interior de cualquiera de ellos. Veámoslo con el ítem alimentarse. En efecto, si problematizamos "alimentarse" según como pueden ser sus articulaciones se puede obtener el cuadro siguiente:

$$
\begin{aligned}
& \text { alimentarse: } \\
& \text { alimentación - mercado de bienes } \\
& \text { dieta - valores y uso de bienes disponibles } \\
& \text { tipo de alimentos - valores y usos } \\
& \text { procedencia - - ubicación espacial del mercado }
\end{aligned}
$$


formas de consumo - relaciones interpersonales en la fam. deseos - orientaciones valorativas - grupos de referencia

Cuando se determina el ítem alimento simultáneamente se está indicando algo acerca de cada una de estas áreas de la realidad: la dieta no es ajena al acceso a los mercados, como tampoco lo es respecto a ciertos valores de uso que se pueden o no transformar en elementos de la dieta. Por su parte, el acceso a los mercados y su distribución espacial implican tipos de relaciones sociales, que, como tales, se transforman en portadores de valores y usos desconocidos; valores y usos que se asocian con determinados grupos sociales. Un ejemplo para aclarar lo que decimos puede ser útil.

Podríamos esquematizar la situación siguiente: dos personas pueden reconocer la misma dieta en proteínas y tener el mismo ingreso. Una de ellas para consumir esa cantidad de proteínas por día requiere ir al almacén para lo cual debe caminar todos los días 250 metros por una calle del pueblo; en cambio la otra para consumir la misma cantidad debe caminar una hora y media hasta el pueblo, y dos horas para regresar a su casa por un sendero en pendiente. Es obvio que los efectos de la misma cantidad de proteínas es diferente en un caso que en otro hasta el límite que sería recomendable que en un caso (el más distante del mercado de abastecimientos) las proteínas fueran reemplazadas por otro tipo de comestibles vegetales. Pero si el patrón cultural de consumo identificado con ciertos grupos sociales, transformados en referentes para la movilidad, como ser los grupos de altos ingresos y urbanos, impedirá que se perciba como aceptable una dieta alternativa que no incluya elementos de la dieta de esos grupos. Es el caso de la carne que en muchas comunidades rurales es expresión de prestigio.

Así como el indicador "comer carne todos los días" no es, en un caso, reflejo de alto nivel de vida, ni menos de racionalidad en el comportamiento para satisfacer las necesidades que aseguren su reproducción como fuerza de trabajo en igualdad de situaciones. En cambio, "no comer carne sino ciertos vegetales" puede ser un indicador más directo de un alto nivel de vida cuando media la racionalidad del individuo de saber optar por alternativas en la satisfacción de sus necesidades.

Si nos detuviéramos en forma más exhaustiva en este análisis, podríamos determinar las opciones de políticas que pueden definirse según si el indicador es leído o no en el contexto de sus articulaciones; articulaciones que no derivan de modelos teóricos, ya que en la realidad pueden ser de tal complejidad que difícilmente puede una teoría prefigurarlas. Es mucho más simple que la construcción de un modelo teórico; pero más complejo que limitarnos a la realidad aludida por el indicador en términos de sus propiedades explícitas. Así, en el ejemplo, el mismo concepto de lo que significa alto o bajo nivel de vida aparece cuestionado: ya no es suficiente con relacionarlo con ciertos elementos nutricionales (para mantenernos dentro de los límites del ejemplo), sino que debe reenfocarse desde el ángulo del máximo aprovechamiento de su asimilación dada la estruc- 
tura de relaciones que caracterizan a la actividad del consumo entendida como acceso a un mercado.

Desde una perspectiva más amplia, yendo más allá de los efectos del consumismo, surgen muchos otros problemas que, relacionados con el modelo económico, configuran situaciones problemáticas no susceptibles de reducirse a una lista de indicadores. Reflexionaremos en una de ellas como es la militarización del Estado.

El hecho básico que enfrentamos es la transformación de las Fuerzas Armadas (en algunos países), la nueva clase política encargada de la administración del Estado y de la representación de los intereses de una fracción de la burguesía como es la financiera. Es preciso distinguir entre la función de administradores de la función del Estado y el carácter del proyecto político y económico con el que se identifican los militares. Distinción que lleva a la pregunta siguiente: ¿es posible que los militares se identifiquen con un proyecto político diferente? Solamente una caracterización de los militares podrá permitirnos responder a la pregunta, a saber:

a) origen social

b) formación ideológica

c) relaciones con la sociedad civil (si se cumplen o no funciones no militares, si se vive en barrios reservados a los militares o no)

d) percepción de la carrera militar por los mismos militares

e) disciplina y verticalidad en la institución militar (si constituyen un cuerpo suficientemente cohesionado para actuar institucionalmente); etc.

La información anterior sobre los militares, analizada desde la distinción entre los militares como administradores y los militares identificados con un proyecto político y económico, reviste un significado diferente. Pues una situación son los militares en el Estado y otra los militares como el Estado. En el primer caso, se hacen cargo de la administración de políticas predeterminadas, mientras que en el segundo determinan sus propias políticas.

El significado de cada uno de los elementos que caracteriza a los militares será diferente según se trate de los militares como administradores o como clase politica, en el sentido siguiente: como administradores no requieren ser autónomos de otros intereses sociales, hecho que ocurre cuando se conceptualizan como clase política.

Si analizamos la información mencionada como necesaria para reconstruir el perfil de los militares desde esta problemática, resulta lo siguiente: la disciplina y verticalidad en la institución puede ser condición de cierta eficiencia dada una cierta formación ideológica civilista de los militares. Pero, si ésta es anticivilista y las relaciones con la sociedad son escasas, la verticalidad interna puede ser la condición para transformarse en clase política. Lo que quiere decir es que la misma información sobre los militares se especifica en su contenido y significación en situaciones concretas como las señaladas: ser administradores o clase política siendo la información la misma. 
Es indiscutible que una caracterización $u$ otra tiene implicaciones políticas diferentes: a) en tanto administradores, la alternativa debe ser definida en relación con los grupos sociales que recurren a los militares, pudiendo éstos reconocer un margen para identificarse con otros proyectos políticos y económicos; y b) como clase política, la alternativa debe ser construida en relación a los militares mismos que sostienen un proyecto propio. La misma información (a, b, c, d, e) permite pasar desde la caracterización de un grupo profesional especializado en determinadas funciones a reconstruir los rasgos de una nueva clase política emergente (situación b).

Este razonamiento problematizador se podría hacer con todas las áreas de la vida social (vida pública, sometimiento social e ideológico, violencia, actividad política, participación en las decisiones, etc.). Sin embargo, en esta oportunidad nos hemos limitado a un ejercicio de reflexión con el propósito de señalar que para que los indicadores puedan cumplir una función en la definición de políticas (a través de sus correspondientes diagnósticos), debe hacerse el esfuerzo de especificarlos según el razonamiento orientado a tratarlos articuladamente. 